\title{
Rib 4
}

National Cancer Institute

\section{Source}

National Cancer Institute. Rib 4. NCI Thesaurus. Code C52764.

The fourth rib counting from the top of the rib cage down. 